\title{
Dynamic quasi-energy-band modulation and exciton effects in biased superlattices driven by a two-color far-infrared field: Disappearance of dynamic localization
}

\author{
Kenta Yashima, Ken-ichi Hino, and Nobuyuki Toshima \\ Institute of Materials Science, University of Tsukuba, 1-1-1 Tennodai, Tsukuba, Ibaraki 305-8573, Japan
}

(Received 28 May 2003; revised manuscript received 31 July 2003; published 23 December 2003)

\begin{abstract}
A theoretical study of the optical and electronic properties of semiconductor superlattices in ac-dc fields, termed the dynamic Wannier-Stark ladder (DWSL), is done. The biased superlattices are driven by two farinfrared fields with different frequencies and relative phase of $\delta$. Here, the frequency of the first laser is equal to the Bloch frequency $\omega_{B}$ of the system under study, while that of the second laser is equal to $2 \omega_{B}$. Quasienergies of the DWSL are calculated based on the Floquet theorem, and the associated linear photoabsorption spectra are evaluated. For $\delta=0$, a gourd-shaped quasi-energy structure characteristic of both dynamic localization (DL) and delocalization (DDL), similar to the usual DWSL driven by a single laser, appears. By changing the ratio of the two laser strengths, however, the width of the quasi-energy band and the locations of both DL and DDL vary noticeably. As for $\delta \neq 0$, on the other hand, band collapse and the associated DL do not necessarily follow. In fact, DL vanishes and the quasi-energy degeneracy is lifted in a certain range of $\delta$. Just DDL remains over the entire range of the laser strength, eventually resulting in a plateaulike band structure in the linear absorption spectra. The basic physics underlying this phenomenon, which can be readily interpreted in terms of a closed analytical expression, is that all quasi-energies for given crystal momenta are out of phase with each other as a function of laser strength without converging to a single point of energy. This is a feature of this DWSL which sharply distinguishes it from a conventional DWSL generated using a single laser to drive it. Furthermore, an exciton effect is incorporated with the above noninteracting problem, so that exciton dressed states are formed. It is found that this effect gives rise to more involved quasi-energy structures and a more pronounced release of the energy degeneracy of DL, leading again to the formation of a band structure in the absorption spectra. These are exclusively due to unevenly spaced exciton energy levels.
\end{abstract}

DOI: 10.1103/PhysRevB.68.235325

PACS number(s): 78.67.Pt

\section{INTRODUCTION}

Since the early studies on Bloch oscillation, ${ }^{1}$ Zener tunneling, ${ }^{2}$ and the Wannier-Stark ladder (WSL), ${ }^{3}$ the problem of a Bloch particle under an external field in an optical or semiconductor superlattices has been thoroughly investigated. ${ }^{4}$ Specifically, quantum systems exposed to strong time-dependent fields provide a variety of intriguing phenomena, such as dynamic localization (DL) and band collapse, ${ }^{5-10}$ the dynamic Franz-Keldysh effect, ${ }^{11-13} \mathrm{THz}$ radiation $^{14-16}$ and $\mathrm{THz}$ photocurrent resonance ${ }^{17}$ in combined static and $\mathrm{THz}$ fields, inverse Bloch oscillation, ${ }^{18,19}$ complex energy spectra in interacting WSL resonance, ${ }^{4}$ and chaotic scattering, ${ }^{4,20}$ all of which are in conjunction with photon-assisted tunneling (PAT). ${ }^{21,22}$ Incorporation of an excitonic effect allows further enrichment of the physics underlying driven quantum systems. For instance, an excitonic intraband polarization is prolonged for much longer than the intraband dephasing time ${ }^{16}$ and the exciton effect in timeintegrated four-wave mixing results in a shift of the DL-peak position, with accompanying extra peaks and shoulders. ${ }^{23}$

A biased superlattice driven by a far-infrared field, which we shall discuss below, is usually called a dynamic WSL (DWSL). ${ }^{24}$ There is an analogy, the well-known Hofstadter problem, ${ }^{25}$ between the quasi-energy spectra of a DWSL and the energy spectra of a Bloch electron in a constant magnetic field, because of the existence of a certain matching ratio of the system parameters in each case. ${ }^{24}$ In the former, the elec- tric matching ratio $\gamma$, represents the ratio of two fundamental space-time areas, $2 \pi d / \omega$ and $h / e F_{0}$, where $F_{0}, d$, and $\omega$ are the static electric field, the superlattice period, and the frequency of the far-infrared field, respectively, and $h$ and $e$ are the Plank constant and the elementary electric charge. In the latter, the magnetic matching ratio is given by the ratio of the two fundamental areas, $l^{2}$ and $h c / e B$, where $B, l$, and $c$ are the constant magnetic field, the lattice constant, and the speed of light, respectively. Note that both the electric and magnetic matching ratios play significant roles in governing the localization behavior in each problem and the corresponding energy spectra result in a self-similar structure.

To the best of our knowledge, DL in periodic motion under the influence of a sinusoidal electric field (without a static electric field) was first discussed by Dunlap and Kenkre, ${ }^{5}$ who pointed out that the localization occurs when the special relation of $J_{0}(e F d / \hbar \omega)=0$ is satisfied, where $F$ is the strength of the ac-field and $J_{0}$ is a zeroth-order Bessel function of the first kind with $\hbar=h / 2 \pi$. Later, Holthaus ${ }^{6}$ showed that the quasi-energy as a function of $e F d / \hbar \omega$ is a repeated gourd shape, and band reformation and band collapse are accompanied by dynamic delocalization (DDL) and DL, respectively. Furthermore, $\mathrm{Zak}^{9}$ pointed out that a similar property also holds in the DWSL and that DL occurs when $J_{n}(e F d / \hbar \omega)=0$, where $n$ is an integer equal to the electric matching ratio

$$
\gamma=\omega_{B} / \omega
$$


where $\omega_{B}=e F_{0} d / \hbar$ is the Bloch frequency of the system concerned, and $J_{n}$ is a $n$ th-order Bessel function of the first kind.

In a DWSL with $\gamma=n$, the WSL energy states of adjacent sites (wells) are strongly coupled to each other due to PAT through a resonant transition, and hence conspicuous band reformation with a large bandwidth is realized around the DDL positions. In the case of a DWSL where $\gamma=p / q$ is a rational number and $p$ and $q$ are prime numbers, ${ }^{24,26}$ the WSL states of each $(q-1)$ th site interact and the nearestneighboring sites no longer couple to each other, leading to a reduction in the band structure and a narrower bandwidth. In the extreme limit of $q \rightarrow \infty$ the bandwidth is zero and all the features in the quasi-energy spectra are just due to DL. Despite the superficial difference between each type of DWSL, i.e., $\gamma$ being an integer or a fraction, the physics underlying them is basically the same and is fully explained by the magnitude of the hopping matrix elements modulated by an external laser field. The spectra of DWSL's have recently been observed in the optical lattices of ultracold atoms, ${ }^{27-32}$ where two counterpropagating laser beams were used to form a standing wave corresponding to the superlattices and where either a tunable frequency difference between these counterpropagating waves or even gravity introduced a constant force corresponding to $e F_{0}$. Incidentally, it is worth noting that there is a similar phenomenon to DL in nature, called the coherent destruction of tunneling. ${ }^{22,33}$ An appropriately designed coherent $\mathrm{cw}$-drive can bring the coherent tunneling between two locally stable wells to an almost complete standstill. This arises from the crossing of two states comprising the tunneling doublet.

To the best of our knowledge, all the studies on DWSL's have thus far been devoted to WSL's driven by a single farinfrared field. One is tempted to see what happens to a DWSL under the influence of a couple of simultaneous farinfrared fields, and how the results thus obtained are altered by the exciton effect. In this paper, a two-color far-infrared field $F(t)$ at time of $t$ is introduced to the system, where the frequencies of the first and the second monochromatic lasers, denoted by $\omega_{1}$ and $\omega_{2}$, are set equal to $\omega_{B}$ and $2 \omega_{B}$ with strengths of $F_{10}$ and $F_{20}$, respectively:

$$
F(t)=\sum_{j=1,2} F_{j}(t)
$$

with

$$
F_{j}(t)=F_{j 0} \cos \left(\omega_{i} t+\delta_{j}\right),
$$

and $\delta_{1}=0$ and $\delta_{2}=\delta$. Accordingly the respective electric matching ratios $\gamma_{j}=\omega_{B} / \omega_{j}$ are given by $\gamma_{1}=1$ and $\gamma_{2}$ $=1 / 2$. Moreover, it is understood that there is a phase difference $\delta$ between these two lasers. Intuitively, one can speculate that in this system the properties of DWSL's with $\gamma$ $=\gamma_{1}$ and $\gamma_{2}$ coexist, and as a result the bandwidths of the quasi-energy spectra attributable to DDL are enlarged to some extent due to the twofold character of PAT, where one way is dominated by the coupling of each adjacent site of the WSL and the other is dominated by the coupling between every other site. As will be shown later, in fact, the spectra
TABLE I. Summary of acronyms used in the text in alphabetical order and the corresponding meanings.

\begin{tabular}{lc}
\hline \hline Acronyms & Meanings \\
\hline DDL & Dynamic delocalization \\
DL & Dynamic localization \\
DVR & Discrete variable representation \\
DWSL & Dynamic WSL \\
EX-DWSL & Excitonic DWSL \\
EX-WSL & Excitonic WSL \\
NI-DWSL & Noninteracting DWSL \\
PAT & Photon-assisted tunneling \\
WSL & Wannier-Stark ladder \\
\hline \hline
\end{tabular}

are rather more involved and the bandwidths are sensitive to the ratio of $F_{20}$ to $F_{10}$ and the relative phase $\delta$. In particular, in a certain range of $\delta$ the DL, which characterizes the DWSL in single-laser driving, vanishes entirely. This is in contrast to the conventional understanding that the DL always manifests itself in a DWSL driven by a single laser. In order to distinguish a DWSL without an exciton effect from the one with it, we shall use acronyms NI-DWSL (noninteracting DWSL) for the former case and EX-DWSL (excitonic DWSL) for the latter. Overall, the primary feature, i.e., the disappearance of DL, is still preserved in an EX-DWSL, though the resulting quasi-energy-band structure is blurred to a certain extent from that of the corresponding NI-DWSL. The linear absorption spectra of the present system obtained by introducing a weak probe field serves to deepen the understanding of the EX-DWSL.

This paper is organized as follows. Methods of calculating the excitonic quasi-energy spectra and the associated linear spectra are given in Sec. II. The results and discussion are in Sec. III. In the first half of this section the basic properties of DWSL's driven by a single laser are reviewed, taking the exciton effect into account. This is instructive for understanding both the similarity to and the difference from the two-color laser driven DWSL discussed in the latter half, where the exciton effect is also addressed. In addition to the results of these numerical calculations, an analytical expression for a two-color laser driven NI-DWSL is derived in order to explore the underlying physics leading to the disappearance of DL. In a straightforward extension, an analytical solution for a DWSL driven by a multicolor laser is also given and discussed. The conclusion is given in Sec. IV. An Appendix is also included to supplement the text. To help the reader, all acronyms defined in this paper are summarized in Table I. Semiconductor superlattices of undoped $45 \AA$ GaAs $/ 45 \AA \mathrm{Al}_{0.3} \mathrm{Ga}_{0.7} \mathrm{As}$ are adopted as samples here and the sinusoidal electric field $F$ as well as the static one $F_{0}$ is introduced along the crystal growth direction (the $z$ axis) to form the DWSL. A schematic view of the present DWSL system is depicted in Fig. 1. Atomic units are used throughout unless otherwise stated.

\section{METHOD OF CALCULATIONS}

\section{A. Excitonic quasi-energy spectra}

First a set of coordinates $\left\{\boldsymbol{\rho}, z_{e}, z_{h}\right\}$ is defined for the present system with $\boldsymbol{\rho}=(|\boldsymbol{\rho}|, \phi)$ representing the in-plane 


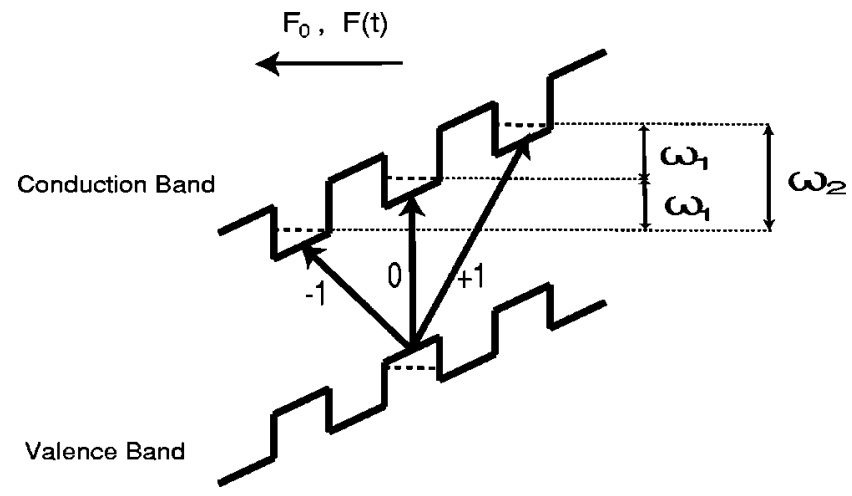

FIG. 1. A schematic diagram of the geometry of the DWSL under study. The static electric field $F_{0}$ and the time-dependent field $F(t)$ are applied along the crystal growth direction. The resonant transitions mediated by $\omega_{1}$ and $\omega_{2}$ are depicted. These give rise to a DWSL with $\gamma=\gamma_{1}$ and $\gamma_{2}$, respectively. When a weak laser probe is introduced, a vertical transition $\nu=0$ and oblique transitions $\nu$ $= \pm 1$ occur, where $\nu$ is the WSL index.

relative vector between an electron $e$ and a hole $h$ with the in-plane angular coordinate $\phi$, and $z_{e}$ and $z_{h}$ being the $z$ coordinates of $e$ and $h$, respectively. Here the center-of-mass motion of an exciton in the plane of the layer is removed. Moreover valence-band mixing is neglected and, for simplicity, the contribution of light holes is omitted, so that just the $s$-radial symmetry of the heavy-hole exciton is taken into account. The complete Hamiltonian $H$ is given by

$$
H\left(\boldsymbol{\rho}, z_{e}, z_{h}, t\right)=\mathcal{H}_{w s l}\left(\boldsymbol{\rho}, z_{e}, z_{h}\right)+F(t)\left(z_{e}-z_{h}\right),
$$

where $\mathcal{H}_{w s l}$ represents the effective-mass Hamiltonian of the exciton WSL (EX-WSL) given by

$$
\mathcal{H}_{w s l}\left(\boldsymbol{\rho}, z_{e}, z_{h}\right)=-\frac{1}{2 m_{\|}} \nabla_{\boldsymbol{\rho}}^{2}+V\left(\boldsymbol{\rho}, z_{e}-z_{h}\right)+h_{w s l}\left(z_{e}, z_{h}\right)
$$

and the second term represents the dipole interaction between $e$ and $h$ with $F$ in the $z$ direction. In Eq. (5) $m_{\|}$is the in-plane reduced mass of $e$ and $h$, and $V$ is the Coulomb potential between them expressed as

$$
V\left(\boldsymbol{\rho}, z_{e}-z_{h}\right)=-\frac{1}{\epsilon \sqrt{\boldsymbol{\rho}^{2}+\left(z_{e}-z_{h}\right)^{2}}},
$$

where $\epsilon$ is the static dielectric constant of the medium. In addition $h_{w s l}$ represents the Hamiltonian of the WSL for a combined subband of $e$ and $h$ and is defined by

$$
h_{w s l}\left(z_{e}, z_{h}\right)=\sum_{j=e, h} h_{w s l}^{(j)}\left(z_{j}\right)
$$

Here

$$
h_{w s l}^{(j)}\left(z_{j}\right)=-\frac{1}{2 m_{z}^{(j)}} \frac{\partial^{2}}{\partial z_{j}^{2}}+u_{j}\left(z_{j}\right) \pm F_{0} z_{j},
$$

where it is understood that the sign in front of the last term, which indicates the dipole interaction of a particle $j$ with $F_{0}$ applied in the $z$ direction is + for $e$ and - for $h$. Moreover, $m_{z}^{(j)}$ is a mass of the particle $j$ in the $z$ direction and $u_{j}$ is the periodic potential of the associated superlattices for the motion of $j$.

The time-dependent Schrödinger equation for the EXDWSL is given by

$$
\left[i \frac{\partial}{\partial t}-H\left(\boldsymbol{\rho}, z_{e}, z_{h}, t\right)\right] \Psi\left(\boldsymbol{\rho}, z_{e}, z_{h}, t\right)=0 .
$$

Because of the periodicity of $F(t)$, namely, $F(t+T)$ $=F(t)$, where $T$ is the period of the system defined as $T$ $=2 \pi / \omega$ with $\omega=\min \left(\omega_{1}, \omega_{2}\right)$ and in light of Floquet's theorem, ${ }^{34}$ we look for a particular solution of the form

$$
\Psi\left(\boldsymbol{\rho}, z_{e}, z_{h}, t\right)=\exp (-i E t) \psi\left(\boldsymbol{\rho}, z_{e}, z_{h}, t\right) .
$$

Here $E$ is a conserved quantity denoting a quasienergy, and $\psi$ is a periodic function satisfying the relation that

$$
\psi\left(\boldsymbol{\rho}, z_{e}, z_{h}, t+T\right)=\psi\left(\boldsymbol{\rho}, z_{e}, z_{h}, t\right) .
$$

The general solution of Eq. (9) is given by a superposition of the Floquet states of Eq. (10), upon which suitable initial conditions can be imposed. The equation of motion for $\psi$ is cast into the form

$$
\left[i \frac{\partial}{\partial t}+E-H\left(\boldsymbol{\rho}, z_{e}, z_{h}, t\right)\right] \psi\left(\boldsymbol{\rho}, z_{e}, z_{h}, t\right)=0 .
$$

In accordance with the relation in Eq. (11), $\psi$ can be expanded as

$$
\psi\left(\boldsymbol{\rho}, z_{e}, z_{h}, t\right)=\sum_{n} \exp (-i n \omega t) \Phi_{n}\left(\boldsymbol{\rho}, z_{e}, z_{h}\right)
$$

Here the basis set $\left\{\Phi_{n}\right\}$ must satisfy the following equation:

$$
\begin{aligned}
\sum_{n^{\prime}}[ & \left\{n \omega+E-\mathcal{H}_{w s l}\left(\boldsymbol{\rho}, z_{e}, z_{h}\right)\right\} \delta_{n n^{\prime}} \\
& \left.-\mathcal{F}_{n n^{\prime}}\left(z_{e}-z_{h}\right)\right] \Phi_{n^{\prime}}\left(\boldsymbol{\rho}, z_{e}, z_{h}\right)=0,
\end{aligned}
$$

where

$$
\mathcal{F}_{n n^{\prime}}=\frac{1}{T} \int_{0}^{T} d t \exp \left[i\left(n-n^{\prime}\right) \omega t\right] F(t) .
$$

In order to seek solutions of Eq. (14), it is convenient to introduce a basis set $\left\{\phi_{\lambda}\right\}$, which is obtained by solving the Schrödinger equation of the EX-WSL:

$$
\left[\mathcal{H}_{w s l}-\mathcal{E}_{\lambda}\right] \phi_{\lambda}\left(\boldsymbol{\rho}, z_{e}, z_{h}\right)=0,
$$

where $\mathcal{E}_{\lambda}$ is the $\lambda$ th energy of the WSL. Expanding $\Phi_{n}$ once again with respect to this basis set as 


$$
\Phi_{n}\left(\boldsymbol{\rho}, z_{e}, z_{h}\right)=\sum_{\lambda} \phi_{\lambda}\left(\boldsymbol{\rho}, z_{e}, z_{h}\right) C_{\lambda n}
$$

yields the following algebraic equation for $\left\{C_{\lambda n}\right\}$ :

$$
\begin{aligned}
& \sum_{\lambda^{\prime} n^{\prime}} {\left[\left(n \omega+E-\mathcal{E}_{\lambda}\right) \delta_{n n^{\prime}} \delta_{\lambda \lambda^{\prime}}-\mathcal{F}_{n n^{\prime}}\left\langle\phi_{\lambda}\left|z_{e}-z_{h}\right| \phi_{\lambda^{\prime}}\right\rangle\right] } \\
& \quad \times C_{\lambda^{\prime} n^{\prime}}=0,
\end{aligned}
$$

with $\langle\cdots\rangle$ denoting integration over $\boldsymbol{\rho}, z_{e}$, and $z_{h}$. This is feasible by means of a standard diagonalization procedure.

It is supposed that the EX-WSL state is formed under a two-subband approximation (a single-subband-pair approximation) in which just the lowest-energy subband pair, composed of the lowest subband of $e$ and the highest subband of $h$, is incorporated. Higher-energy pairs are neglected. Moreover, Zener tunneling and the WSL resonance ${ }^{4}$ are considered as minor effects, where the latter is relevant to tunneling through tilted barriers of WSL into continuum states with a finite resonance width, namely, the shape resonance. These assumptions are warranted in the present system, as is shown later. In addition, excitonic Fano resonance and the related multichannel nature are also ignored, though all of the EXWSL states are related to such resonance states as distinct from pure bound states, and this effect plays a significant role in more accurate and quantitative level of investigation. $^{35-39}$

With following the approximations made above, the EXWSL wave function is written as

$$
\phi_{\lambda}\left(\boldsymbol{\rho}, z_{e}, z_{h}\right)=\sum_{\mu} \chi_{\mu}\left(z_{e}, z_{h}\right) \mathcal{G}_{\mu \lambda}(\boldsymbol{\rho}) .
$$

Here $\chi_{\mu}$ is the $\mu$ th subband wave function of a combined WSL given by

$$
\chi_{\mu}\left(z_{e}, z_{h}\right)=\frac{1}{\sqrt{N_{z}}} \sum_{\nu} \exp \left(i \nu K_{z} d\right) \xi_{\mu+\nu}^{(e)}\left(z_{e}\right) \xi_{\nu}^{(h)}\left(z_{h}\right)
$$

where $\xi_{\nu}^{(e(h))}$ is the $\nu$ th WSL-subband wave function of $e(h)$ satisfying the Schrödinger equation for $h_{w s l}^{(e(h))}$, and $K_{z}$ is the center-of-mass momentum in the $z$ direction. In fact $K_{z}$ is set zero here, since it does not affect photoabsorption spectra. ${ }^{40,41} N_{z}$ is the number of periods of the superlattices included in the calculations. Furthermore, $\mathcal{G}_{\mu \lambda}$ in Eq. (19) is defined as

$$
\mathcal{G}_{\mu \lambda}(\boldsymbol{\rho})=\frac{1}{\sqrt{2 \pi}} \exp \left(\operatorname{im}_{\mu} \phi\right) \sum_{l}|\boldsymbol{\rho}|^{m} \varphi_{l}(|\boldsymbol{\rho}|) a_{\mu l \lambda},
$$

where $m_{\mu}$ is the in-plane angular moment pertinent to the subband $\mu$ and is set to zero because only the $s$-radial symmetry is considered. The discrete variable representation (DVR) is employed for the set of basis functions $\left\{\varphi_{l}\right\}$ and hence the subscript $l$ refers the $l$ th abscissa of the Gaussian quadrature. For details of DVR, consult Refs. 42-46. Utiliz- ing this recipe enables one to greatly reduce the computational burden of seeking the solutions $\left\{\phi_{\lambda}\right\}$ of Eq. (16) with high accuracy owing to the DVR ansatz

$$
\int d|\boldsymbol{\rho}| \varphi_{l}(|\boldsymbol{\rho}|) \mathcal{V}_{\mu \nu}(|\boldsymbol{\rho}|) \varphi_{l^{\prime}}(|\boldsymbol{\rho}|) \approx \mathcal{V}_{\mu \nu}\left(\left|\boldsymbol{\rho}_{l}\right|\right) \delta_{l l^{\prime}}
$$

where $\mathcal{V}_{\mu \nu}$ is an effective potential defined by

$$
\mathcal{V}_{\mu \nu}(|\boldsymbol{\rho}|)=\int d z_{e} d z_{h} \chi_{\mu}^{*}\left(z_{e}, z_{h}\right) V\left(\boldsymbol{\rho}, z_{e}-z_{h}\right) \chi_{\nu}\left(z_{e}, z_{h}\right)
$$

$\left\{a_{\mu l \lambda}\right\}$ is the set of unknown coefficients to be determined.

The remaining task is to obtain the WSL-subband wave functions $\xi_{\nu}^{(j)}$ for $j=e, h$ used in Eq. (20), satisfying

$$
\left[h_{w s l}^{(j)}\left(z_{j}\right)-\varepsilon_{\nu}^{(j)}\right] \xi_{\nu}^{(j)}\left(z_{j}\right)=0,
$$

where the energy is given by $\varepsilon_{\nu}^{(j)}=\varepsilon_{0}^{(j)}+\nu \omega_{B}$ with the WSL index denoted by $\nu$. Because of the two-subband approximation used here, the subband index to be attached to $\xi_{\nu}^{(j)}$ and $\varepsilon_{\nu}^{(j)}$ is uniquely given and then omitted. This wave function is further expressed by the superposition of the wave functions $\eta_{k}^{(j)}$ of the associated superlattices with the Bloch momentum $k=2 \pi n / N_{z} d$ for $n=-N_{z} / 2,-N_{z} / 2+1, \ldots, N_{z} / 2$ $-2, N_{z} / 2-1\left(N_{z}\right.$ is even) in the following equation:

$$
\xi_{\nu}^{(j)}\left(z_{j}\right)=\sum_{k} \eta_{k}^{(j)}\left(z_{j}\right) b_{k \nu}^{(j)}
$$

where

$$
\eta_{k}^{(j)}\left(z_{j}\right)=\frac{1}{\sqrt{N_{z}}} \sum_{l m} \exp (i k l d) B_{m+N_{b s} l, \kappa}\left(z_{j}\right) c_{m}^{(j)},
$$

and $\left\{b_{k \nu}^{(j)}\right\}$ and $\left\{c_{m}^{(j)}\right\}$ are sets of expansion coefficients. Here $B_{i, \kappa}(z)$ represents the $i$ th normalized basis-spline ( $B$-spline) function of the order $\kappa .{ }^{47}$ The advantages of employing such a piecewise basis set are described below. The knot sequence for the set of $B$ splines ranges over the whole superlattice crystal, and it is understood that $N_{b s}$ knots are introduced per unit cell so as to satisfy the relation

$$
B_{i, \kappa}(z+n d)=B_{i-N_{b s} n, \kappa}(z),
$$

where $n$ an integer. This ensures the Bloch theorem for $\eta_{k}^{(j)}$, that is,

$$
\eta_{k}^{(j)}\left(z_{j}+n d\right)=\exp (i k n d) \eta_{k}^{(j)}\left(z_{j}\right)
$$

In Fig. 2, the wave function $\xi_{0}^{(e)}$ is depicted along with the geometry of the present WSL for a typical value of $F_{0}=11$ $\mathrm{kV} / \mathrm{cm} . \xi_{\nu}^{(e)}$ is readily obtained from $\xi_{\nu}\left(z_{e}\right)=\xi_{0}\left(z_{e}-\nu d\right)$. It is clearly found that the wave function is well localized within just a couple of sites and the tails of it are negligibly small. This justifies the above-mentioned approximation of neglecting the WSL resonance. Furthermore, disregarding Zener tunneling was justified numerically by comparing the energy obtained within the present single-subband-pair ap- 


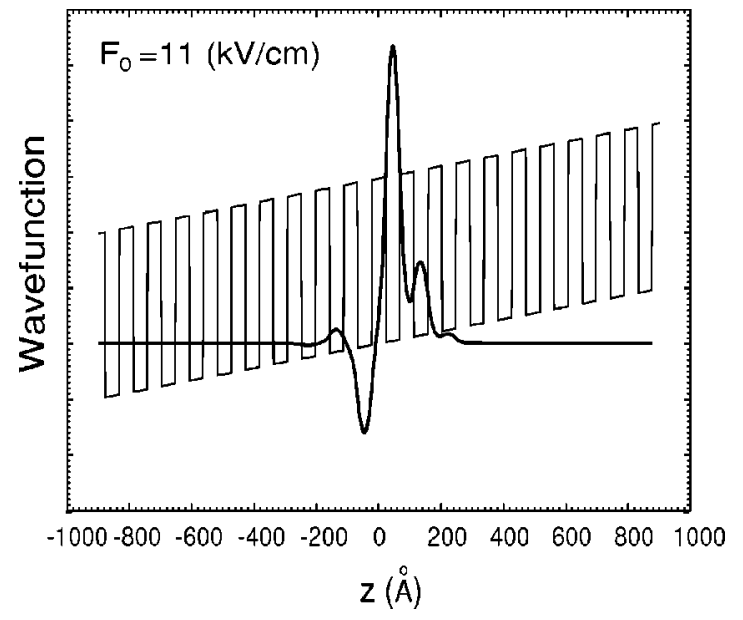

FIG. 2. The EX-WSL wave function of $\xi_{0}^{(e)}$ for $F_{0}$ $=11 \mathrm{kV} / \mathrm{cm}$. The geometry of the present WSL is also depicted.

proximation with that evaluated by including the two lowestenergy subband pairs. The latter shifts just slightly from the former and therefore the contribution from Zener tunneling is considered to be minor. However, it should be remarked that for a still larger $F_{0}$ both the WSL resonance and Zener tunneling would be more significant so that a more sophisticated description of the WSL would be required.

Conventionally, with the single-subband-pair approximation, each $\xi_{\nu}^{(j)}$ and $\varepsilon_{\nu}^{(j)}$ is given one by one in terms of the Kane representation in conjunction with the Kronig-Penny model for superlattices with rectangular periodic potentials. ${ }^{41,48}$ Practically, this recipe is limited to the squarewell potential and the situation in which multiple subband pairs are not strongly coupled. On the other hand, the present $B$-spline method is more flexible and can be applied to any type of potential, regardless of whether this is smooth or discontinuous. In fact, for the rectangular potentials with which we are concerned here, this method allows one to evaluate a large number of eigenvalues with high accuracy at one stroke by introducing knot multiplicity ${ }^{47}$ to the positions where the potential is discontinuous, i.e., at the boundaries between the wells and the barriers. With minor changes, the same computer program can be readily applied to, for instance, a sinusoidal potential that models the WSL of optical superlattices. ${ }^{27-32}$ Furthermore, it is straightforward to apply this to the more formidable problems of the Zener tunneling when $F_{0}$ is relatively strong. The extension of this method to other systems is beyond the scope of the present paper, and it will be discussed elsewhere.

\section{B. Linear absorption spectra}

The EX-DWSL state is probed by introducing a weak monochromatic test laser with a vector potential e $A_{p} \exp$ $\left(-i \omega_{p} t\right)$ under the dipole approximation, where $A_{p}$ is the amplitude of the laser, $\mathbf{e}$ is the polarization vector assumed here to be in the plane of the layer (TE polarization), and $\omega_{p}$ is the frequency. Time-dependent perturbation theory is used to find the dipole-transition amplitude of the $\alpha$ th excited state, $\Psi_{\alpha}^{(c v)}(\mathbf{r}, t)$, with a quasienergy $E_{\alpha}$ in the remote future $(t \rightarrow \infty)$ and this is expressed as

$$
b_{\alpha}(t=\infty)=-i \mathbf{e} \cdot A_{p} \int_{0}^{\infty} d t \mathbf{D}_{\alpha 0}(t) \exp \left(i\left[E_{\alpha}-E_{0}-\omega_{p}\right] t\right) .
$$

Here the $\alpha$ th state is excited from the ground state of crystal, $\Psi_{0}(\mathbf{r})$, which has energy $E_{0}$ at $t=0$. Moreover, the dipole moment of the transition is given by

$$
\mathbf{D}_{\alpha 0}(t)=\left\langle\Psi_{\alpha}^{(c v)}(\mathbf{r}, t)|\mathbf{p}| \Psi_{0}(\mathbf{r})\right\rangle,
$$

where $\mathbf{r}$ is a group of the position vectors of all the participating electrons, $\mathbf{p}=-i \boldsymbol{\nabla}_{\mathbf{r}}$, and the $t$ dependence of the excited DWSL state is explicitly represented in $\mathbf{D}_{\alpha 0}$ and $\Psi_{\alpha}^{(c v)}$.

As usual, $\Psi_{0}$ is expressed by the Slater determinant of the valence-band Wannier functions $\left\{a_{\mathbf{R}}^{(v)}(\mathbf{r})\right\},{ }^{49}$ where $\mathbf{R}$ is the crystal position given here by $\mathbf{R}=\left(\boldsymbol{\rho}, z_{e}, z_{h}\right)$ with the unimportant center-of-mass motion in the plane of the layer being omitted, as stated in Sec. II A. $\Psi_{\alpha}^{(c v)}$ is cast in the form ${ }^{49}$

$$
\Psi_{\alpha}^{(c v)}(\mathbf{r}, t)=\frac{1}{\sqrt{N}} \sum_{\mathbf{R}} \psi_{\alpha}(\mathbf{R}, t) \Lambda_{\mathbf{R}}^{(c v)}(\mathbf{r}),
$$

where $\Lambda_{\mathbf{R}}^{(c v)}$ is also given in terms of the Slater determinant composed of $\left\{a_{\mathbf{R}}^{(v)}(\mathbf{r})\right\}$ and the conduction-band Wannier functions $\left\{a_{\mathbf{R}}^{(c)}(\mathbf{r})\right\}, N$ is the total number of lattices concerned, and the envelope function $\psi_{\alpha}$ is the same as that already defined in Eqs. (10) and (11), but with the label $\alpha$ added. Putting $\Psi_{0}$ and $\Psi_{\alpha}^{(c v)}$ into Eq. (30) yields

$$
\mathbf{D}_{\alpha 0}(t)=\frac{1}{\sqrt{N}} \sum_{\mathbf{R}} \psi_{\alpha}^{*}(\mathbf{R}, t) \mathbf{d}_{\mathbf{R}}^{(c v)} \approx \frac{1}{\sqrt{N}} \mathbf{d}_{\mathbf{0}}^{(c v)} v_{\alpha}(t)
$$

where

$$
\mathbf{d}_{\mathbf{R}}^{(c v)}=\left\langle a_{\mathbf{R}}^{(c)}(\mathbf{r})|\mathbf{p}| a_{\mathbf{0}}^{(v)}(\mathbf{r})\right\rangle
$$

and

$$
v_{\alpha}(t)=\int d z \psi_{\alpha}^{*}(\boldsymbol{\rho}=\mathbf{0}, z, z, t)
$$

The second equality in Eq. (32) is due to the fact that $\mathbf{R}=\mathbf{0}$ is dominant for dipole-allowed transitions. Therefore Eq. (29) can be recast as

$$
\begin{aligned}
b_{\alpha}^{\mathcal{K}}(\infty)= & -i\left(\mathbf{e} \cdot \mathbf{d}_{\mathbf{0}}^{(c v)}\right) \frac{A_{p}}{\sqrt{N}} \sum_{l=0}^{N_{T}} \int_{l T}^{(l+1) T} d t v_{\alpha}(t) \\
& \times \exp \left(i\left[E_{\alpha}-E_{0}-\omega_{p}\right] t\right) \\
= & -i\left(\mathbf{e} \cdot \mathbf{d}_{\mathbf{0}}^{(c v)}\right) \frac{A_{p} T}{\sqrt{N}} \sum_{l} \\
& \times \exp \left(i\left[E_{\alpha}-E_{0}-\omega_{p}-\mathcal{K} \omega\right] l T\right) M_{\alpha}^{\mathcal{K}} \\
= & -2 \pi i \delta\left(E_{\alpha}-E_{0}-\omega_{p}-\mathcal{K} \omega\right)\left(\mathbf{e} \cdot \mathbf{d}_{\mathbf{0}}^{(c v)}\right) \frac{A_{p}}{\sqrt{N}} M_{\alpha}^{\mathcal{K}} .
\end{aligned}
$$



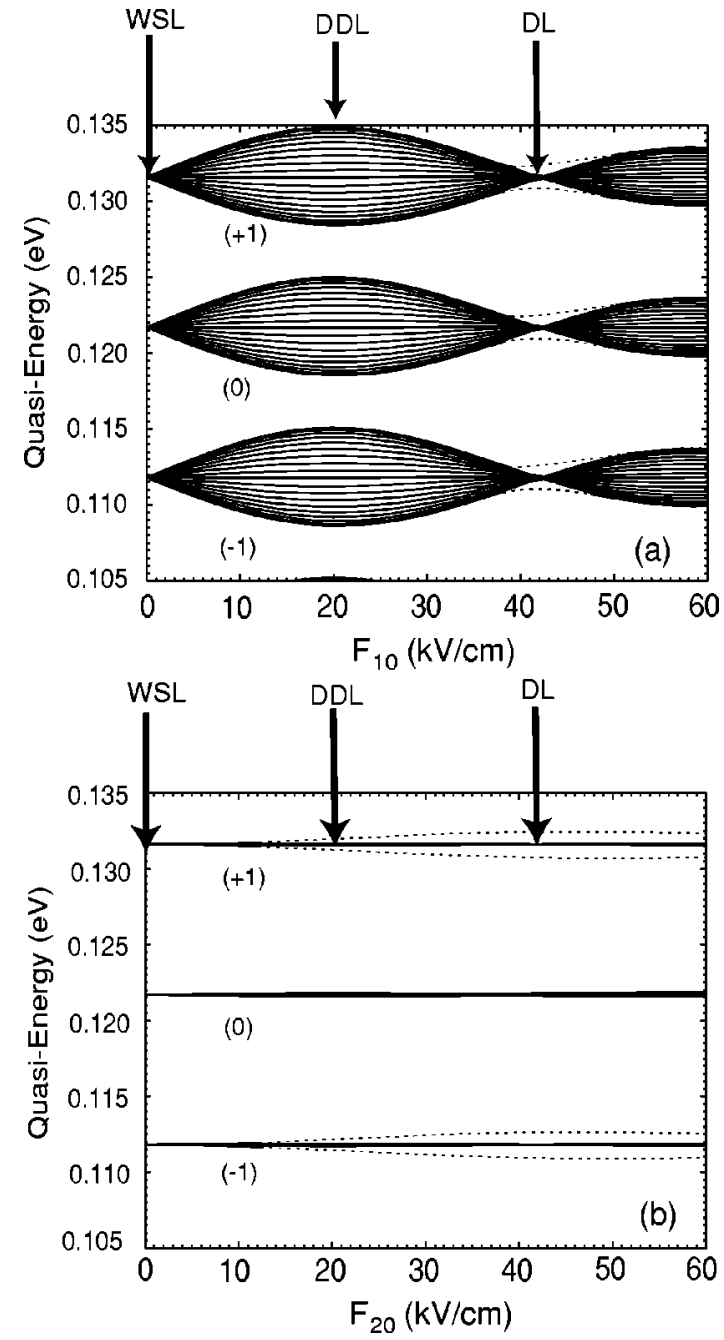

FIG. 3. (a) Quasi-energies for a NI-DWSL driven by a single laser with $\gamma=\gamma_{1}$ as a function of $F_{10}$. The positions of WSL, DDL, and DL are specified by arrows. The numbers in parentheses represent the parent band $\nu_{b}=0$ and the photon sidebands $\nu_{b}= \pm 1$. Dashed lines indicate the numerical errors incurred by incorporation of a finite number of superlattice sites $\left(N_{z}=20\right)$ in the calculations. (b) The same as (a) but for a NI-DWSL with $\gamma=\gamma_{2}$ as a function of $F_{20}$.

In the first equality, the whole $t$ domain is divided into $N_{T}$ pieces each with an interval $T$ and assuming $N_{T} \rightarrow \infty$. In the second equality, the relation of Eq. (11) has been employed. Moreover, $\mathcal{K}(\neq 0)$ refers to the number of the photon sideband (replica) relevant to the exciton Floquet state $\alpha$ and $\mathcal{K}=0$ corresponds to the parent band. $M_{\alpha}^{\mathcal{K}}$ is of the form

$$
M_{\alpha}^{\mathcal{K}}=\frac{1}{T} \int_{0}^{T} d t \exp (i \mathcal{K} \omega t) v_{\alpha}(t)=\int d z \Phi_{\alpha}^{*} \mathcal{K}(\boldsymbol{\rho}=\mathbf{0}, z, z) .
$$

For the second equality, Eq. (13) has been used. Thus $\mathcal{K}$ is identical to the number of dressing-field photons participating in the formation of the state $\alpha$. The $\mathcal{K}$ dependence plays the role of Umklapp processes in the temporally periodic system.
The transition probability $P_{\alpha}$ to $\alpha$ per unit time and unit cell ends up as

$$
\begin{aligned}
P_{\alpha}= & \sum_{\mathcal{K}} \frac{\left|b_{\alpha}^{\mathcal{K}}(\infty)\right|^{2}}{N_{T} T}=2 \pi\left|\left(\mathbf{e} \cdot \mathbf{d}_{\mathbf{0}}^{(c v)}\right)\right|^{2} \frac{A_{p}^{2}}{N} \\
& \times \sum_{\mathcal{K}} \delta\left(E_{\alpha}-E_{0}-\omega_{p}-\mathcal{K} \omega\right)\left|M_{\alpha}^{\mathcal{K}}\right|^{2} .
\end{aligned}
$$

The absorption coefficient $\alpha\left(\omega_{p}\right)$ is, needless to say, proportional to the expression in the above equation. Note that similar expression is given in Ref. 24, however, without derivation nor with the exciton effect.

\section{RESULTS AND DISCUSSION}

The present section consists of DWSL's generated by a single dressing field and by a two-color dressing field. It is assumed that the contribution of the bound states of the WSL exciton to the expansion given in Eq. (17) dominate and the contribution from the continuum states is negligibly small. Inspection of the linear absorption spectra of the EX-WSL, which shows that the continuum contribution is usually much smaller than that of the bound state, implies that this is a reasonable assumption and the continuum contribution is unimportant unless the Fano resonance and particular effects of the continuum are taken into account. ${ }^{35,36,39}$ This assumption is undoubtedly desirable in order that the spectra of the EX-DWSL can be compared with those of the NI-DWSL on an equal footing in terms of the corresponding quasi-energy curves in the $E-F_{j 0}$ plane as is shown below. If all the contributions of the continuum are incorporated in Eq. (17), the quasi-energies become homogeneously distributed over the whole plane with the measure zero, that is, within the zero distance between the nearest-neighbor quasi-energy positions. ${ }^{50}$ In this case, it would no longer be possible to understand the EX-DWSL on an equal footing with the NIDWSL. In the present calculations, a couple of the lowestlying exciton bound states pertaining to each WSL site are incorporated. The energy levels of states with larger WSL indices become evenly-spaced and identical to the associated subband energies. Hereafter, the setup parameters $N_{z}=20$ and $F_{0}=11 \mathrm{kV} / \mathrm{cm}$ are adopted for the calculations of the DWSL.

\section{A. Single-laser driving}

This section presents the basic properties of DWSL's driven by lasers $F_{1}(t)$ and $F_{2}(t)$, taking account of the exciton effect. The results of the NI-DWSL are shown in Fig. 3. where the parent band is labeled $\nu_{b}=0$ and the photon sidebands are labeled $\nu_{b} \neq 0$. At $F_{j 0}=0(j=1,2)$, this label is equivalent to the WSL index. The NI-DWSL with $\gamma=\gamma_{1}$ in Fig. 3(a) is shown by the gourd-shaped structure of the quasienergy $E$ as a function of $F_{10}$, and the repetitive behavior of both DDL and DL are evident with increasing $F_{10}$. By use of the nearest-neighbor tight-binding model for a superlattice miniband 


$$
\varepsilon(k)=E_{0}-\frac{\Delta}{2} \cos (k d)
$$

with the energy of the band center $E_{0}$ and bandwidth $\Delta$, the quasi-energy can be obtained from a single-cycle average of the instantaneous energy $\varepsilon[k-(1 / c) A(t)]$ :

$$
E_{\nu_{b}}(k)=\frac{1}{T} \int_{0}^{T} d t \varepsilon\left(k-\frac{1}{c} A(t)\right)+\nu_{b} \omega_{1},
$$

where $k$ is the Bloch momentum, which is a good quantum number of the system under study, and $A(t)$ is the vector potential associated with the combined ac-dc field. This is readily reduced to the following analytical expression: 6

$$
E_{\nu_{b}}(k)=E_{0}-\frac{\Delta}{2} \cos (k d)(-1)^{n} J_{n}\left(\frac{F_{10} d}{\omega_{1}}\right)+\nu_{b} \omega_{1},
$$

where $n$ is an integer equal to $\gamma_{1}$. As $F_{10}$ increases, first the band structure is retrieved, with a larger bandwidth and showing signs of DDL. The width eventually reaches a maximum, resulting in the most pronounced DDL, and then decreases. Finally, when $F_{10}$ coincides with zeros of $J_{n}$, the band collapses, leading to the formation of a knot between adjacent lobes, and DL is realized concomitantly with it. Such a tendency is repeated. It is evident that this phenomenon arises exclusively from PAT.

Figure 3 (b) shows the quasi-energy structure resulting from the NI-DWSL with $\gamma=\gamma_{2}$. It is readily seen that the bandwidth is much reduced compared with the NI-DWSL with $\gamma=\gamma_{1}$, and the DDL character seems to almost vanish due to the reduced magnitude of the hopping matrix elements. Moreover, two bands appear at an interval of $\omega_{2}$ $=2 \omega_{B}$ corresponding to the Brillouin zone of the temporal periodic system. These observations are similar to those demonstrated by the nearest-neighbor tight-binding model. ${ }^{24}$ As noted in Sec. I, the same basic physics governed simply by the magnitude of the hopping matrix elements modulated by an external laser field, underlies the two DWSL's with $\gamma=\gamma_{1}$ and $\gamma_{2}$.

The properties of the NI-DWSL are confirmed by examining the associated linear absorption spectra depicted in Fig. 4, which were calculated based on Eq. (37). ${ }^{51}$ Figures $4(\mathrm{a}-\mathrm{e})$ show the spectra of the NI-DWSL with $\gamma=\gamma_{1}$ at five typical field strengths relevant to the positions of WSL, DDL, and DL. It is found that contributions from the photon sidebands, $\nu_{b}= \pm 1$, are always less significant than the contribution from the parent band $\nu_{b}=0$. Hence, just the parent band is considered. At the DDL positions, the spectral patterns are reminiscent of the spectra of superlattices characterized by the appearance of a plateau with the $M_{0}$ and $M_{1}$ Van Hove singularities. ${ }^{52}$ The maximum bandwidth of the leftmost lobe is $6.5 \mathrm{meV}$ which is reduced by about $40 \%$ of the width of the original superlattices of $11.2 \mathrm{meV}$. The width of DDL seems less dependent on $F_{0}$. On the other hand, at the DL positions, a single sharp peak appears in place of the plateau, where the peak height is enhanced in comparison with that of the WSL in Fig. 4(a), due presumably to an increase in the density of states ascribable to the quasi-energy degeneracy.

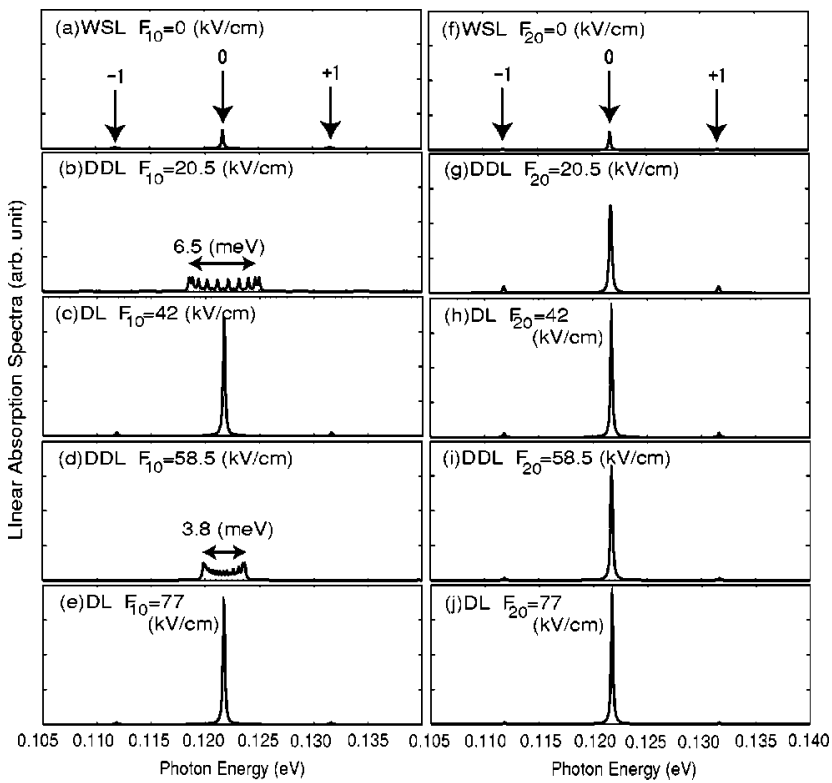

FIG. 4. Linear absorption spectra of a NI-DWSL with (a)-(e) $\gamma=\gamma_{1}$ and (f) $-(\mathrm{j}) \quad \gamma=\gamma_{2}$ corresponding to Fig. 3 for several typical field strengths as a function of the irradiated photon energy $\omega_{p}$ measured from the bottom of the conduction band. The vertical arrow indicates the WSL index. The horizontal double arrow with a number attached indicates the bandwidth for DDL. The spectra are convoluted with the Lorentzian profile with a homogeneous broadening of $0.2 \mathrm{meV}$ to aid the presentation.

In Figs. 4(f-j), the spectra of the NI-DWSL with $\gamma=\gamma_{2}$ are shown at the same field strengths as above. No DDL spectral pattern is observed, and all spectra feature the DL spectral pattern with an enhancement effect similar to Figs. 4(c) and 4(e).

As for the exciton effect on these DWSL's, the quasienergies corresponding to Fig. 3 are shown in Fig. 5, where the horizontal arrows indicate the isolated quasi-energy traces of the exciton bound states, labeled as $\nu_{b}(n s)$. Overall, for the EX-DWSL with $\gamma=\gamma_{1}$, in Fig. 5(a), the gourdshaped structure is once again obtained. Aside from this, some curves of the low-lying exciton bound states are found isolated from the main spectra, which are ascribable to the higher-lying Rydberg states of exciton. The quasi-energy degeneracy is lifted to a certain extent at the DL positions due to the unevenly spaced exciton levels, resulting in rather broadened spectra. The profile of the parent band is duplicated exactly in every replica due to $\omega_{1}=\omega_{B}$. In Fig. 5(b), which shows the EX-DWSL with $\gamma=\gamma_{2}$, partial removal of the degeneracy is also seen. However, the profile of the parent band is different from the adjacent photon sidebands due to $\omega_{2}=2 \omega_{B}$.

The linear absorption spectra corresponding to Fig. 4 are shown in Fig. 6. Both EX-DWSL's, with $\gamma=\gamma_{1}$ and $\gamma_{2}$, are dominated by strong exciton peaks ascribable to the dressed $0(1 s)$ state. Moreover, it is found that every peak height of this state is enhanced by the dressing effect from the associated WSL regardless of the DDL and DL positions. ${ }^{53}$ However, the band structure can still be perceived at the DDL positions of Figs. 6(b) and 6(d), even though they are smaller 

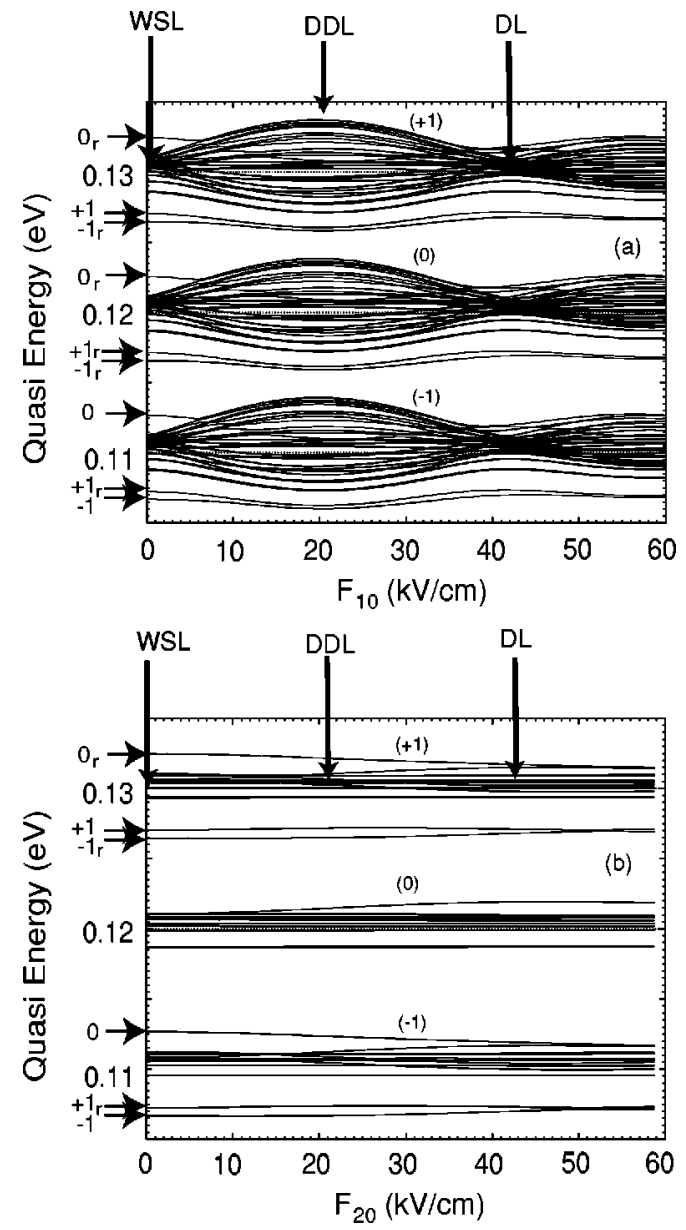

FIG. 5. The same as Fig. 3 but for an EX-DWSL with (a) $\gamma$ $=\gamma_{1}$ and (b) $\gamma=\gamma_{2}$. The numbers specified by horizontal arrows represent the quasi-energy positions of isolated dressed exciton states, where $\nu=0$, and \pm 1 refer to the $0(1 s)$ and $\pm 1(1 s)$ states, respectively, and the symbols with the subscript $r, \nu_{r}$, refer to the replicas of the states labeled $\nu$. The numbers in parentheses represent the parent band, $\nu_{b}=0$, and the photon sidebands, $\nu_{b}= \pm 1$.

than the exciton peaks. The variance of the binding energy of the dressed states versus $F_{j 0}$ is shown in Fig. 7. The energy is evaluated from the difference between the quasi-energies of the EX-DWSL in Fig. 5 and the NI-DWSL in Fig. 3. The trace of the EX-DWSL with $\gamma=\gamma_{1}$ in Fig. 7(a) shows that the binding energy of the $0(1 s)$ state gradually increases with stronger $F_{10}$, accompanied by oscillations with an amplitude of about $1 \mathrm{meV}$. The DL tends to provide larger binding energies than the DDL, which is similar to the tendency observed in the EX-WSL when compared with the corresponding superlattices with $F_{0}=0 .{ }^{40}$ On the other hand, in Fig. 7(b), the dressing effect on the $0(1 s)$ state gives rise to a smooth increase of the binding energy of the EX-DWSL with $\gamma=\gamma_{2}$ and the dressing effect on the $-1(1 s)$ state gives rise to a smooth decrease of the binding energy.

\section{B. Two-color laser driving}

Now we analyze the dressing effect due to a two-color laser beam composed of $F_{1}(t)$ and $F_{2}(t)$ with a relative

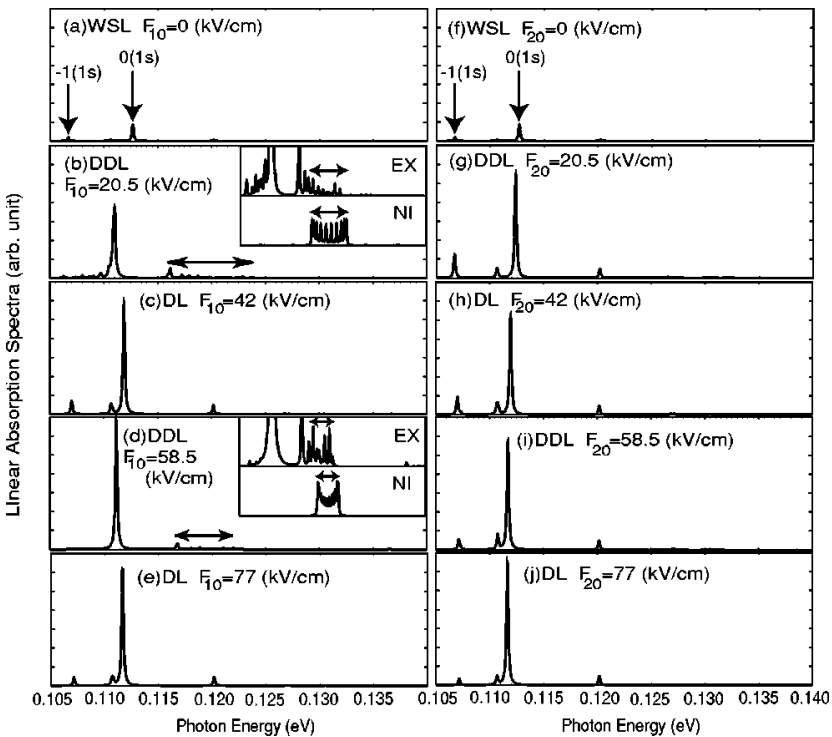

FIG. 6. The same as Fig. 4 but for an EX-DWSL with (a)-(e) $\gamma=\gamma_{1}$ and (f) $-(\mathrm{j}) \gamma=\gamma_{2}$, corresponding to Fig. 5. The upper insets of (b) and (d) are enlarged views of the respective main panels for comparison with the spectra of the corresponding NI-DWSL depicted in the lower insets. The horizontal double arrow represents the bandwidth for DDL.

phase $\delta$. The quasi-energies of the NI-DWSL and EXDWSL and the corresponding linear absorption spectra are presented first based on the full numerical calculations. Next, in order to deepen our understanding of the results thus ob-

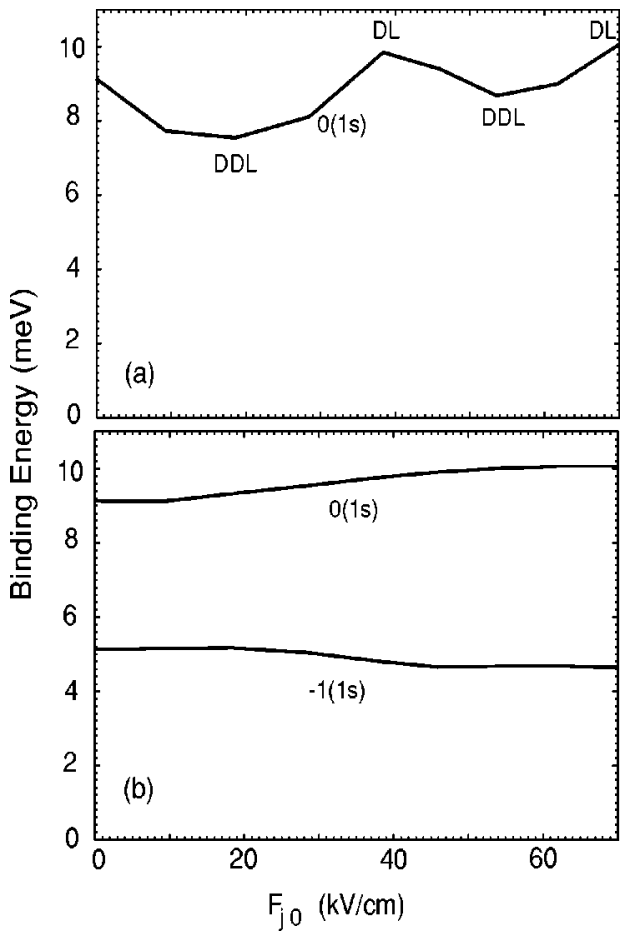

FIG. 7. Binding energies of the dressed exciton states as a function of $F_{j 0}(j=1,2)$, which were evaluated from Figs. 3 and 5. (a) The $0(1 s)$ state for an EX-DWSL with $\gamma=\gamma_{1}$. (b) The $0(1 s)$ and $-1(1 s)$ states for an EX-DWSL with $\gamma=\gamma_{2}$. 


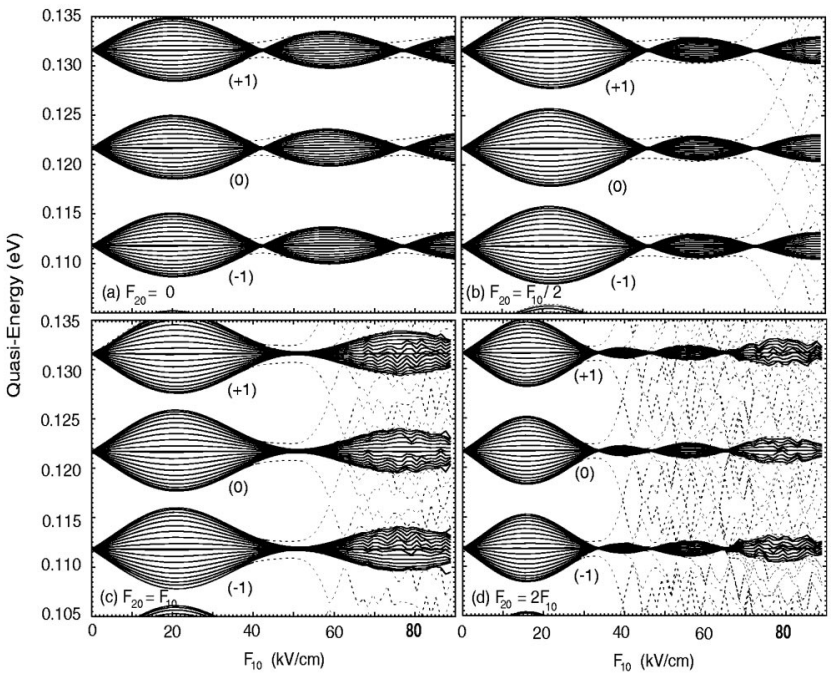

FIG. 8. Quasi-energies for a NI-DWSL driven by a two-color laser with $\delta=0$ as a function of $F_{10}$, for four typical values of $\Gamma$ $=F_{20} / F_{10}$ equal to (a) 0 , (b) $1 / 2$, (c) 1 , and (d) 2 . The numbers in parentheses represent the parent band $\nu_{b}=0$ and the photon sidebands $\nu_{b}= \pm 1$. The ripples, denoted by dashed lines, which are seen, especially, in the higher-field regions of (b), (c), and (d), are numerical errors incurred in part by incorporation of a finite number of superlattice sites $\left(N_{z}=20\right)$ in the calculations, and in part by the limited capacity of the memory and CPU time in the workstation utilized here.

tained, we also conduct an analysis based on the nearestneighbor tight-binding model, similar to the analysis done for single-laser driving.

\section{Numerical analysis}

To begin with, the quasi-energy of the NI-DWSL with $\delta$ $=0$ is discussed, based on the calculated results of Fig. 8, which shows the variance of the profiles with respect to the ratio $\Gamma=F_{20} / F_{10}$. It can clearly be seen that spectral widths and locations of the DL and the maximum DDL vary significantly depending on $\Gamma$. More specifically, the bandwidth of the first (leftmost) lobe tends to increase by a couple of meV when $\Gamma=1 / 2$ and 1 [Figs. 8(b) and $8(\mathrm{c})$ ]. The widths of the leftmost lobes are $6.5,7.8,8.4$, and $7.4 \mathrm{meV}$ for $\Gamma$ $=0,1 / 2,1$, and 2 , respectively. On the other hand, the widths of the second and the third lobes are clearly reduced, especially when $\Gamma=2$ [Fig. $8(\mathrm{~d})]$. Note that, along with these features, the DL is still evident for $\delta=0$.

Figure 9 shows the quasi-energy curves for different $\delta$ 's with $\Gamma$ fixed to $1 / 2$ corresponding to Fig. 8(b). In comparison with Fig. 9 (a) where $\delta=0$, it is readily seen that the knots between the lobes become blurred and as a result every DL disappears in Figs. 9(b) and 9(c). Following extended numerical calculations, it was found that this tendency in the leftmost knot is maximized in the vicinity of $\delta=\pi / 4$. Thus far the appearance of DL with sharp knots is considered to be one of the unique characteristics of a DWSL, irrespective of whether $\gamma$ is equal to either $\gamma_{1}$ or $\gamma_{2}$. Nevertheless, it is evident that, with the present two-color laser dressing, this statement cannot always be applied over a certain range of $\delta$,

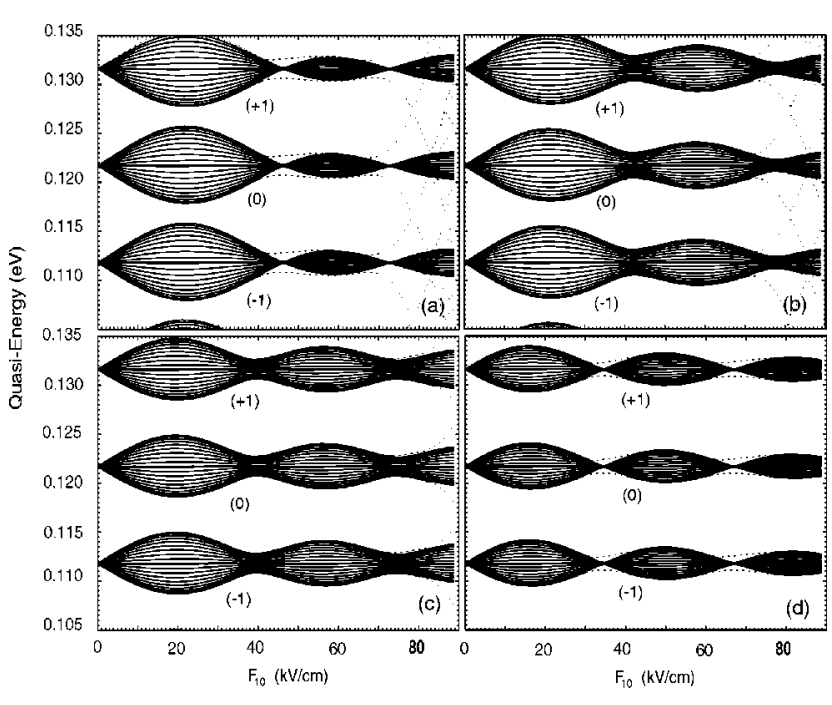

FIG. 9. Quasi-energies for a NI-DWSL driven by a two-color laser with $\Gamma=1 / 2$ as a function of $F_{10}$, for four typical values of $\delta$ equal to (a) 0 , (b) $\pi / 4$, (c) $\pi / 2$, and (d) $\pi$. The numbers in parentheses and the ripples have the same meaning as in Fig. 8.

and hence it is limited to the case of the single-laser DWSL. For $\delta$ greater than $\pi / 2$, the disappearance becomes less pronounced, and finally for $\delta=\pi$ [Fig. 9(d)], DL is retrieved, however, with a much reduced bandwidth around the maximum-DDL positions. Furthermore, the profile of the quasi-energy curves remain unaltered with respect to the phase inversion whereby $\delta$ is replaced by $\delta^{\prime}=-\delta$. This is easily demonstrated by the time-reversal relation, $\psi_{\delta^{\prime}}^{*}(t)$ $=\exp (i \sigma) \psi_{\delta}(-t)$, where $\psi_{\delta}$ and $\psi_{\delta^{\prime}}$ are wave functions satisfying Eq. (12) for the relative phases of $\delta$ and $\delta^{\prime}$, respectively, and $\sigma$ is an undetermined phase factor. This operation ensures the invariance of $E$.

The quasi-energy structure of the EX-DWSL generated by the two-color laser beam, which corresponds to the NIDWSL with $\delta=\pi / 4$ and $\Gamma=1 / 2$ in Fig. 9(b). is shown in Fig. 10 . It can be seen that the DL blurs and vanishes more definitely than in the NI-DWSL due to the additional effect of

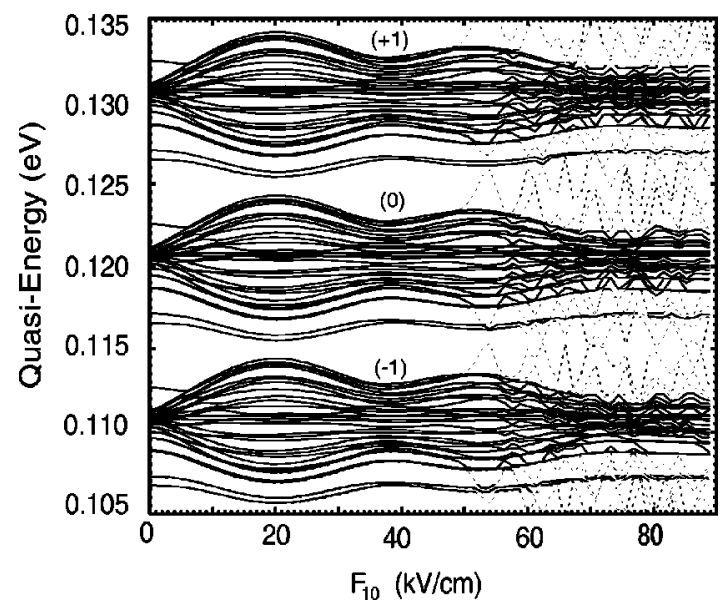

FIG. 10. Quasi-energies for an EX-DWSL driven by a two-color laser with $\delta=\pi / 4$ and $\Gamma=1 / 2$ as a function of $F_{10}$. The numbers in parentheses and the ripples have the same meaning as in Fig. 8. 


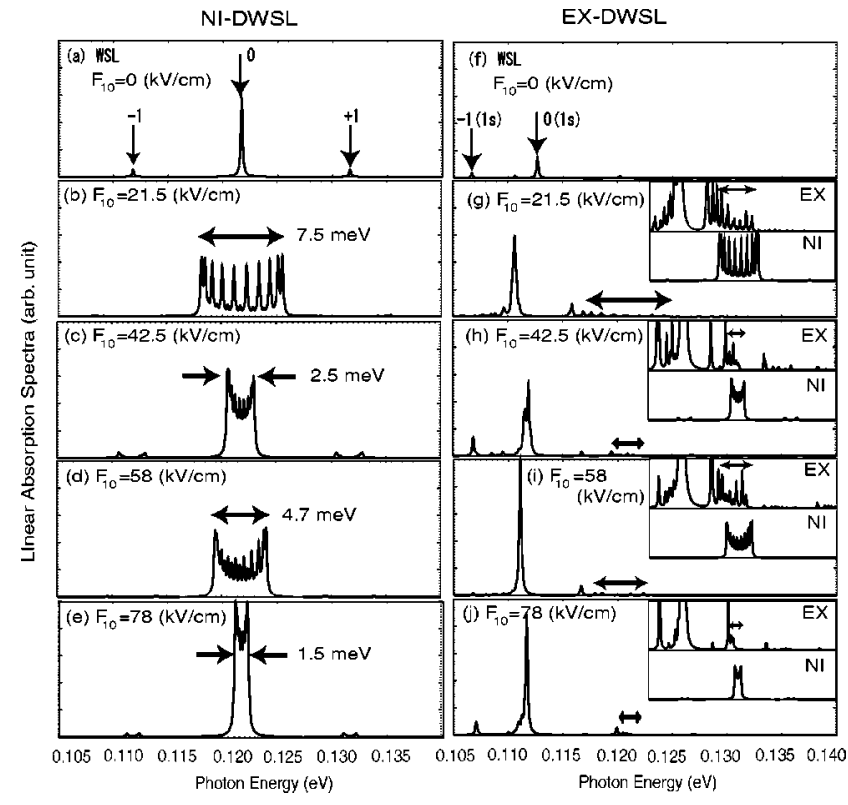

FIG. 11. Linear absorption spectra corresponding to Figs. 9(b) and 10 for several typical field strengths as a function of the irradiated photon energy $\omega_{p}$ measured from the bottom of the conduction band. The vertical arrow indicates the WSL index and WSL exciton states. (a)-(e) NI-DWSL and (f)-(j) EX-DWSL. The upper insets in $(\mathrm{g})-(\mathrm{j})$ are enlarged views of the respective main panels for comparison with the spectra of the corresponding NI-DWSL depicted in the lower insets. The horizontal double arrow represents the bandwidth for DDL. The spectra are convoluted with the Lorentzian profile having a homogeneous broadening of $0.2 \mathrm{meV}$ to aid the presentation.

the unevenly aligned energy levels of the exciton. Depicted in Figs. 11(a-e) and $11(\mathrm{f}-\mathrm{j})$ are the linear absorption spectra of the NI-DWSL and EX-DWSL generated by the two-color laser dressing, which are relevant to the quasi-energy traces of Figs. 9(b) and 10, respectively. Around the dim DL positions of Figs. 11(c) and 11(e), an obvious band structure characterized by plateaus with Van Hove singularities appears. The same is also true of Figs. 11(h) and 11(j), except that the spectra are more or less modulated by the exciton effect. In fact the peaks due to the exciton bound states are so strong that the plateau structures are liable to be concealed. This tendency is also common to the well-known exciton absorption spectra of superlattices which have a series of sharp peaks of exciton bound states followed by a plateau attributed to continuum states. Note that the spectral enhancement effect that makes the DWSL exciton peak considerably greater than the corresponding WSL peak is still present.

Incidentally, it seems that the overall shapes of the lobes and the isolated bands of Fig. 10 look similar to those in Fig. 5(a) for the EX-DWSL caused by single-laser dressing in the sense that both spectra around the DL positions are blurred due to the exciton effect. However, it should be noted that in the former the dim appearance of the DL results in a quasienergy band structure [see Figs. 11(h) and 11(j)], while in the latter it causes the formation of just a single peak without any band structure [see Figs. 6(c) and 6(e)]. This distin- guishes the DWSL generated by two-color laser driving from the single-laser DWSL. It can be concluded that the disappearance of DL in the present case originates from the nature of the NI-DWSL rather than that of the EX-DWSL, and that the exciton effect plays a subsidiary role.

\section{Model analysis}

The results of the NI-DWSL in Sec. III B 1 can be interpreted in terms of the analytical expression for the quasienergy given by Eq. (39). In the present case, $A(t)$ is of the form

$$
A(t)=-c\left[F_{0} t+\frac{F_{10}}{\omega_{1}} \sin \left(\omega_{1} t\right)+\frac{F_{20}}{\omega_{2}} \sin \left(\omega_{2} t+\delta\right)\right] .
$$

According to Appendix, the quasienergy is given in a closed analytical form as follows:

$E_{\nu_{b}}(k)=E_{0}+\frac{\Delta}{2} \sum_{l=-\infty}^{\infty} J_{2 l+1}\left(x_{1}\right) J_{l}\left(x_{2}\right) \cos (k d+l \delta)+\nu_{b} \omega_{1}$,

where $x_{j}=F_{j 0} d / \omega_{j} \quad(j=1,2)$. If $F_{20}$ goes to zero, this immediately becomes identical to Eq. (40) for $n=1$. In this context, Eq. (42) is considered as the general expression of Eq. (40).

Now we discuss the $\delta$ dependence of $E_{\nu_{b}}(k)$ in order to understand the numerical results given in Fig. 9 in more depth. First the case of $\delta=n \pi$ ( $n$-integer) is taken into account. Thus Eq. (42) is cast into the form

$$
E_{\nu_{b}}(k)=E_{0}+Z\left(x_{1}, x_{2}\right) \cos (k d)+\nu_{b} \omega_{1},
$$

where

$$
Z\left(x_{1}, x_{2}\right)=\frac{\Delta}{2} \sum_{l=-\infty}^{\infty}(-1)^{n l} J_{2 l+1}\left(x_{1}\right) J_{l}\left(x_{2}\right)
$$

Note that the function $Z$ is independent of $k$, which leads to the important consequence that every $E_{\nu_{b}}(k)$ for a given $k$ takes the same value of $E_{0} \bmod \omega_{1}$ at positions where $Z\left(x_{1}, x_{2}\right)=0$. Such behavior is equivalent to the band collapse observed in the single-laser driving of Sec. III A. The appearance of the DL structure in Fig. 9(a) $(\delta=0)$ and Fig. 9(d) $(\delta=\pi)$ can be precisely understood in terms of Eq. (43). On the other hand, in the case of $\delta \neq n \pi$, the quasienergy given by Eq. (42) is no longer reduced to the form of Eq. (43). Hence the values of the set of $x_{1}$ and $x_{2}$ at which $E_{\nu_{b}}(k)=E_{0} \bmod \omega_{1}$ are different for each $k$, and this property gives rise to the disappearance of DL.

Practical evaluation of Eq. (42) reveals that the infinite series over $l$ rapidly converges and the degree of precision attained by incorporating at most up to $|l|=3$ or 4 is to the tenth digit. Therefore, for the qualitative discussion below, $E_{\nu_{b}}(k)$ can be well approximated by taking only the first few terms. That is, 


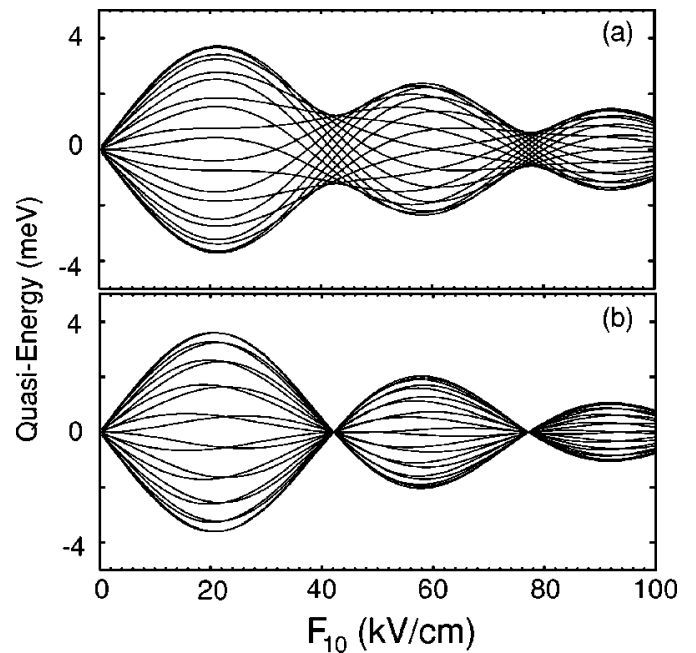

FIG. 12. Analytical evaluation of the quasi-energies of Eq. (45) for a NI-DWSL driven by a two-color laser with $\delta=\pi / 4$ and $\Gamma$ $=1 / 2$ as a function of $F_{10}$. (a) Full calculations. (b) Calculations incorporating just the first term in the square brackets of Eq. (45). The quasi-energy is measured from $E_{0}$.

$$
\begin{aligned}
E_{\nu_{b}}(k) \approx & E_{0}+\frac{\Delta}{2}\left[J_{1}\left(x_{1}\right) J_{0}\left(x_{2}\right) \cos (k d)\right. \\
& +J_{3}\left(x_{1}\right) J_{1}\left(x_{2}\right) \cos (k d+\delta) \\
& \left.+J_{1}\left(x_{1}\right) J_{1}\left(x_{2}\right) \cos (k d-\delta)\right]+\nu_{b} \omega_{1} .
\end{aligned}
$$

Figure 12(a) shows the consequently calculated quasi-energy for $\Gamma=1 / 2$ and $\delta=\pi / 4$ with $\nu_{b}=0$, where $\Delta=11.2 \mathrm{meV}$ and $d=90 \AA$, similar to the numerical calculations of Sect. III B 1 . It is readily seen that it does indeed reproduce the result shown in Fig. 9(b), above all, the disappearance of DL. For comparison, the contribution of the first most dominant term in the squared brackets of Eq. (45) is shown in Fig. 12(b). It is found that this term gives rise to band collapse, whereas the extra higher-order contributions from the second and third terms, which stand out exclusively in the vicinity of the DL positions, lift the degeneracy of the quasi-energy, leading to the disappearance of DL. In conclusion, the result which asserts that the DL vanishes over the whole range of the laser strength is due to the fact that the quasi-energy for each $k$ oscillates out of phase as a function of $F_{10}$, and the band collapse is considered to be just a special case of accidental in-phase behavior.

Finally, we discuss an analytical solution of the quasienergy driven by a multicolor laser field. Instead of Eq. (41), the vector potential $A(t)$ in this case is given by

$$
A(t)=-c\left[F_{0} t+\sum_{j=1}^{N_{d}} \frac{F_{j 0}}{\omega_{j}} \sin \left(\omega_{j} t+\delta_{j}\right)\right],
$$

where $N_{d}$ represents the number of monochromatic lasers included, and $\delta_{1}$ is assumed to be zero as before. Repeated applications of Eq. (A2) immediately provide the following expression:

$$
\begin{aligned}
E_{\nu_{b}}(k)= & E_{0}-\frac{\Delta}{2} \sum_{l_{2}, \ldots, l_{N_{d}}=-\infty}^{\infty} J_{-N_{T}}\left(x_{1}\right) J_{l_{2}}\left(x_{2}\right) \cdots J_{N_{d}}\left(x_{N_{d}}\right) \\
& \times \cos \left(k d+\sum_{j=2}^{N_{d}} l_{j} \delta_{j}\right)
\end{aligned}
$$

modulo $\omega_{1}$, where $N_{T}=N_{1}+\sum_{j=2}^{N_{d}} N_{j} l_{j}, x_{j}=F_{j 0} d / \omega_{j}$, and it is assumed that $\omega_{j}$ for $j \neq 1$ is a multiple of $\omega_{1}$, that is, $\omega_{j} / \omega_{1}$ is equal to an integer $N_{j}(j \neq 1)$ along with $N_{1}$ $=\omega_{B} / \omega_{1}$. This is a generalization of Eqs. (40) and (42). What is important is that even with multi-color laser driving the basic physics of the disappearance of DL is just the same as for the two-color laser driving case discussed above. That is, DL takes place only in the special case where $\sum_{j=2}^{N_{d}} l_{j} \delta_{j}$ $=n \pi$ ( $n$-integer), and if not, the features of the quasienergy are always due to band formation with a finite width, leading to the delocalization of an electron.

\section{CONCLUSION}

The EX-DWSL of the biased superlattices driven by a two-color far-infrared field with different frequencies, $\omega_{1}$ $=\omega_{B}$ and $\omega_{2}=2 \omega_{B}$, different field strengths $F_{10}$ and $F_{20}$, and relative phase $\delta$ was theoretically investigated based on the Floquet approach for calculating the gourd-shaped quasienergies $E$ and the linear absorption spectra of the system. The notable finding of this study is the disappearance of DL in the whole $E-F_{10}$ plane over a certain range of $\delta$, and this effect is maximized in the vicinity of $\delta=\pi / 4$. In this situation, the lobes of the quasi-energy for NI-DWSL are no longer connected by a sharp knot, and the location of the knot is blurred to some extent. The disappearance of the DL results in the formation of the quasi-energy band structure. In accordance with the analytical expression for the NI-DWSL obtained here, this phenomenon stems from the out-of-phase behavior of each quasi-energy curve for a given $k$ with respect to the change in $F_{10}$, while the band collapse observed in two-color laser driving with $\delta=n \pi$ ( $n$-integer) as well as in single-laser driving is interpreted as a special case of the in-phase behavior. This feature of the two-color laser driven DWSL is also well understood by resorting to the corresponding linear absorption spectra. As a matter of fact, it has thus far been considered that the appearance of DL is one of the peculiar properties of the single-laser driven DWSL. Nevertheless, this is not always correct for two-color laser dressing. The same consequence also holds in the EXDWSL, except that the unevenly spaced exciton energy levels further blur the knot position of the DL and strong absorption peaks ascribable to exciton bound states are revealed along with the band structure.

\section{ACKNOWLEDGMENTS}

This research was partially supported by a Grant-in-Aid for Scientific Research (B) from Japan Society for the Promotion of Science. 


\section{APPENDIX DERIVATION OF Eq. (42)}

In view of Eq. (41) we obtain

$$
e^{-i(1 / c) A(t)}=\left[\sum_{l=-\infty}^{\infty} J_{l}\left(x_{2}\right) e^{i\left\{\left(\omega_{B}+l \omega_{2}\right) t+l \delta\right\}}\right] e^{i x_{1} \sin \left(\omega_{1} t\right)}
$$

by use of the relation

$$
e^{i x_{2} \sin \varphi}=\sum_{l=-\infty}^{\infty} J_{l}\left(x_{2}\right) e^{i l \varphi},
$$

where $\varphi=\omega_{2} t+\delta .^{54}$ Then Eq. (39) is reduced to

$$
\begin{aligned}
E_{\nu_{b}}(k)= & E_{0}-\frac{\Delta}{2} \operatorname{Re}\left[\frac{1}{T} \int_{0}^{T} d t e^{i[k-(1 / c) A(t)] d}\right]+\nu_{b} \omega_{1} \\
= & E_{0}-\frac{\Delta}{2} \operatorname{Re}\left[\sum_{l=-\infty}^{\infty} J_{l}\left(x_{2}\right) e^{i(k d+l \delta)}\right. \\
& \left.\times \frac{1}{2 \pi} \int_{0}^{2 \pi} d \theta e^{i\left\{(2 l+1) \theta+x_{1} \sin \theta\right\}}\right]+\nu_{b} \omega_{1} \\
= & E_{0}+\frac{\Delta}{2} \sum_{l=-\infty}^{\infty} J_{2 l+1}\left(x_{1}\right) J_{l}\left(x_{2}\right) \\
& \times \cos (k d+l \delta)+\nu_{b} \omega_{1} .
\end{aligned}
$$

${ }^{1}$ F. Bloch, Z. Phys. 52, 555 (1928).

${ }^{2}$ C. Zener, Proc. R. Soc. London, Ser. A 145, 523 (1934).

${ }^{3}$ G.H. Wannier, Phys. Rev. 117, 432 (1960).

${ }^{4}$ M. Glück, A.R. Kolovsky, and H.J. Korsch, Phys. Rep. 366, 103 (2002).

${ }^{5}$ D.H. Dunlap and V.M. Kenkre, Phys. Rev. 34, 3625 (1986).

${ }^{6}$ M. Holthaus, Phys. Rev. Lett. 69, 351 (1992).

${ }^{7}$ M. Holthaus and D. Hone, Phys. Rev. B 47, 6499 (1992).

${ }^{8}$ M. Holthaus and D.W. Hone, Phys. Rev. B 49, 16605 (1994).

${ }^{9}$ J. Zak, Phys. Rev. Lett. 71, 2623 (1993).

${ }^{10}$ S. Raghavan, V.M. Kenkre, D.H. Dunlap, A.R. Bishop, and M.I. Salkola, Phys. Rev. A 54, R1781 (1996).

${ }^{11}$ A.P. Jauho and K. Johnsen, Phys. Rev. Lett. 76, 4576 (1996).

${ }^{12}$ K. Johnsen and A.P. Jauho, Phys. Rev. B 57, 8860 (1998).

${ }^{13}$ K.B. Nordstrom, K. Johnsen, S.J. Allen, A.P. Jauho, B. Birnir, J. Kono, T. Noda, H. Akiyama, and H. Sakaki, Phys. Rev. Lett. 81, 457 (1998).

${ }^{14}$ T. Meier, G. von Plessen, P. Thomas, and S.W. Koch, Phys. Rev. B 51, 14490 (1995).

${ }^{15}$ M.M. Dignam, Phys. Rev. B 59, 5770 (1999).

${ }^{16}$ J.M. Lachaine, M. Hawton, J.E. Sipe, and M.M. Dignam, Phys. Rev. B 62, R4829 (2000).

${ }^{17}$ J.B. Xia, Phys. Rev. B 58, 3565 (1998).

${ }^{18}$ K. Unterrainer, B.J. Keay, M.C. Wanke, S.J. Allen, D. Leonard, G. Medeiros-Ribeiro, U. Bhattacharya, and M.J.W. Rodwell, Phys. Rev. Lett. 76, 2973 (1996).

${ }^{19}$ X.G. Zhao, G.A. Georgakis, and Q. Niu, Phys. Rev. B 56, 3976 (1997).

${ }^{20}$ D.V. Savin and V.V. Sokolov, Phys. Rev. E 56, R4911 (1997).

${ }^{21}$ R. Ferreira and G. Bastard, Surf. Sci. 229, 424 (1990).

${ }^{22}$ M. Grifoni and P. Hänggi, Phys. Rep. 304, 229 (1998).

${ }^{23}$ R.B. Liu and B.F. Zhu, Phys. Rev. B 59, 5759 (1999).

${ }^{24}$ X.G. Zhao, R. Jahnke, and Q. Niu, Phys. Lett. A 202, 297 (1995).

${ }^{25}$ D.R. Hofstadter, Phys. Rev. B 14, 2239 (1976).

${ }^{26}$ K.C. Je, S.H. Park, and Y. Kim, Phys. Rev. B 64, 075111 (2001).

${ }^{27}$ Q. Niu, X.-G. Zhao, G.A. Georgakis, and M.G. Raizen, Phys. Rev. Lett. 76, 4504 (1996).

${ }^{28}$ S.R. Wilkinson, C.F. Bharucha, K.W. Madison, Q. Niu, and M.G. Raizen, Phys. Rev. Lett. 76, 4512 (1996).

${ }^{29}$ M. BenDahan, E. Peike, J. Reichel, Y. Castin, and C. Salomon,
Phys. Rev. Lett. 76, 4508 (1996).

${ }^{30}$ M.C. Fischer, K.W. Madison, Q. Niu, and M.G. Raizen, Phys. Rev. A 58, R2648 (1998).

${ }^{31}$ K.W. Madison, M.C. Fischer, R.B. Diener, Q. Niu, and M.G. Raizen, Phys. Rev. Lett. 81, 5093 (1998).

${ }^{32}$ K.W. Madison, M.C. Fischer, and M.G. Raizen, Phys. Rev. A 60, R1767 (1999).

${ }^{33}$ F. Grossmann, T. Dittrich, P. Jung, and P. Hänggi, Phys. Rev. Lett. 67, 516 (1991).

${ }^{34}$ F. H. M. Faisal, Theory of Multiphoton Processes (Plenum Press, New York, 1987), Chap. 10.

${ }^{35}$ D.M. Whittaker, J. Phys. IV 5, 199 (1993).

${ }^{36}$ D.M. Whittaker, Europhys. Lett. 31, 55 (1995).

${ }^{37}$ K. Hino, Phys. Rev. B 62, R10 626 (2000).

${ }^{38}$ K. Hino, Phys. Rev. B 64, 075318 (2001).

${ }^{39}$ K. Hino, K. Goto, and N. Toshima, Phys. Rev. B (to be published).

${ }^{40}$ M.M. Dignam and J.E. Sipe, Phys. Rev. B 43, 4097 (1991).

${ }^{41}$ N. Linder, Phys. Rev. B 55, 13664 (1997).

${ }^{42}$ D.O. Harris, G.G. Engerholm, and W.D. Gwinm, J. Chem. Phys. 43, 1515 (1965).

${ }^{43}$ A.S. Dickinson and P.R. Certain, J. Chem. Phys. 49, 4209 (1968).

${ }^{44}$ J.C. Light, I.P. Hamilton, and J.V. Lill, J. Chem. Phys. 82, 1400 (1985).

${ }^{45}$ J.T. Muckerman, Chem. Phys. Lett. 173, 200 (1990).

${ }^{46}$ For applications to low-dimensional exciton problems, see, $\mathrm{K}$. Hino, J. Phys. Soc. Jpn. 67, 3159 (1998); 71, 2280 (2002); Solid State Commun. 128, 9 (2003), and Refs. 37 and 38.

${ }^{47}$ C. de Boor, A Practical Guides to Splines (Springer, New York, 1978), Chap. 9.

${ }^{48}$ N. Linder, K.H. Schmidt, W. Geisselbrecht, G.H. Döhler, H.T. Grahn, K. Ploog, and H. Schneider, Phys. Rev. B 52, 17352 (1995).

${ }^{49}$ M. Ueta, H. Kanzaki, K. Kobayasi, Y. Toyozawa, and E. Hanamura, Excitonic Processes in Solids, Solid-State Sciences Vol. 60 (Springer, New York, 1986), Chap. 1.

${ }^{50}$ D.W. Hone, R. Ketzmerick, and W. Kohn, Phys. Rev. A 56, 4045 (1997).

${ }^{51}$ For the NI-DWSL, in fact, the absorption spectra of Fig. 4 correspond to $\partial \alpha\left(\omega_{p}\right) / \partial \omega_{p}$, rather than $\alpha\left(\omega_{p}\right)$ in order to make a close comparison with the spectra for the EX-DWSL of Fig. 6. 
This manipulation is also applied to Fig. 11 for the two-color laser driving.

${ }^{52}$ The $M_{0}$ and $M_{1}$ singularities mean the critical points at the bottom and top of the miniband, respectively, following Ref. 36.
${ }^{53}$ P. Ray and P.K. Basu, Phys. Rev. B 50, 14595 (1994).

${ }^{54}$ I. S. Gradshteyn and L. M. Ryzhih, Table of Integrals, Series, and Products, Corrected and Enlarged Edition (Academic Press, New York, 1980), p. 973. 